\title{
RANDOM FIXED POINTS OF WEAKLY INWARD OPERATORS IN CONICAL SHELLS
}

\author{
ISMAT BEG \\ Kuwait University \\ Department of Mathematics \\ P.O. Box 5969 \\ Safat 13060, Kuwait \\ NASEER SHAHZAD \\ Quaid-i-Azam University \\ Department of Mathematics \\ Islamabad, Pakistan
}

(Received February, 1995; Revised March, 1995)

\begin{abstract}
Conditions for random fixed points of condensing random operators are obtained and subsequently used to prove random fixed point theorems for weakly inward operators in conical shells.
\end{abstract}

Key words: Banach Space, Inward Operator, Random Fixed Point.

AMS (MOS) subject classifications:47H10, 60H25, 47H40, 47H04.

\section{Introduction}

The fixed point theorems play a very important role in the questions of existence, uniqueness and successive approximations for various type of equations. The random fixed point theorems are useful tools for solving various problems in the theory of random equations, which form a part of random functional analysis. In recent years, there has been exciting interaction between analysis and probability theory, furnishing a rich source of problems for analysis. Bharucha-Reid [2] proved the random version of Schauder's Fixed Pint Theorem. Random fixed point theory has further developed rapidly in recent years; see e.g. Itoh [4], Sehgal and Waters [8], Sehgal and Singh [7], Papageorgiou [6], Lin [5], Xu [10], Tan and Yuan [9] and Beg and Shahzad [1]. This paper is a continuation of these investigations. We have obtained random fixed points of weakly inward random operators in the shell, a stochastic analogue of the results of Deimling [3].

\section{Preliminaries}

Let $(\Omega, \mathcal{A})$ be a measurable space with $\mathcal{A}$ as a sigma-algebra of subsets of $\Omega$. Let $X$ be a real Banach space; a map $q: \Omega \rightarrow X$ is called measurable if for each open subset $G$ of $X, q^{-1}(G) \in \mathcal{A}$. Let $S$ be a nonempty subset of $X$; a map $f: \Omega \times S \rightarrow X$ is called a random operator if for each fixed $x \in S$, the map $f(\cdot, x): \Omega \rightarrow X$ is measurable. A measurable map $\zeta: \Omega \rightarrow S$ is a random fixed point of the random operator $f$ if $f(\omega, \zeta(\omega))=\zeta(\omega)$ for each $\omega \in \Omega$.

Let $K \subset X$ be a cone; that is, $K$ is closed and convex such that $\lambda K \subset K$ for all $\lambda \geq 0$ and $K \cap(-K)=\{0\}$. Denote $\{x \in K:\|x\| \leq r\}$ by $K_{r}$ and $\{x \in K: \rho \leq\|x\| \leq r\}$ by $K_{\rho, r}$ for 
some $0<\rho<r$. A map $f: K_{r} \rightarrow X$ is $\alpha$-condensing if $f$ is continuous and bounded and $\alpha(f(B))<$ $\alpha(B)$ for all $B \subset K_{r}$ with $\alpha(B)>0$, where $\alpha(B)=\inf \{d>0: B$ can be covered by a finite number of sets of diameter $\leq d\}$. A random operator $f: \Omega \times K_{r} \rightarrow X$ is $\alpha$-condensing if for each $\omega \in \Omega$, $f(\omega, \cdot)$ is $\alpha$-condensing. An operator $f: \Omega \times C \rightarrow X$ is said to be weakly inward on a closed convex subset $C$ of $X$ if for each $\omega \in \Omega, f(\omega, x) \in \overline{I_{c}(x)}$ for all $x \in C$, where $\overline{I_{c}(x)}$ denotes the closure of $\{x+\lambda(y-x): \lambda \geq 0, y \in C\}$. In the case $C=K$, it simply becomes $x \in \partial K$. The latter, $x^{*} \in K^{*}$ (the dual cone), and $x^{*}(x)=0$ imply that

$$
x^{*}(f(\omega, x)) \geq 0
$$

for each $\omega \in \Omega$, where $X^{*}$ is the dual space, $K^{*}=\left\{x^{*} \in X^{*}: x^{*}(x) \geq 0\right.$ on $\left.K\right\}$ and $\partial K$ is the boundary of $K$. Suppose $f: \Omega \times K_{r} \rightarrow X$, satisfies $x \in \partial K,\|x\| \leq r, x^{*} \in K^{*}$ and $x^{*}(x)=0$ imply that $x^{*}(f(\omega, x)) \geq 0$ for each $\omega \in \Omega$. Let $P_{r}: K \rightarrow K_{r}$ be the radial projection; that is, $P_{r}(x)=x$ for $\|x\| \leq r$ and $P_{r}(x)=\frac{r x}{\|x\|}$ for $\|x\|>r$. Then for each $\omega \in \Omega, f(\omega, \cdot) \circ P_{r}$ satisfies (1). Since $\alpha\left(P_{r}(B)\right) \leq \alpha(B)$ for all bounded $B \subset K, f(\omega, \cdot) \circ P_{r}$ is $\alpha$-condensing if $f$ is.

\section{Main Results}

Theorem 1: Let $X$ be a separable Banach space, $K \subseteq X$ a cone and $f: \Omega \times K_{r} \rightarrow X$ an $\alpha$-condensing random operator, such that

(i) $\quad x \in \partial K,\|x\| \leq r, x^{*} \in K^{*}$ and $x^{*}(x)=0$ imply that $x^{*}(f(\omega, x)) \geq 0$ for all $\omega \in \Omega$, and

(ii) $\quad f(\omega, x) \neq \lambda x$ on $\|x\|=r$ and for all $\omega \in \Omega$ and $\lambda>1$, are satisfied.

Then $f$ has a random fixed point.

Proof: Define a random operator $g: \Omega \times K_{r} \rightarrow X$ by $g(\omega, x)=\left(f(\omega, \cdot) \circ P_{r}\right) x$. It is clear from Deimling [3, proof of Theorem 1] that for any $\omega \in \Omega, g(\omega, \cdot)$ is $\alpha$-condensing and weakly inward on $K_{r}$ for some $r>0$. Further application of Xu [10, Theorem 2] or Tan and Yuan [9, Corollary $2.6]$ gives the desired result.

Theorem 2: Let $X$ be a separable Banach space, $K \subset X$ a cone, $f: \Omega \times K_{r} \rightarrow X$ an $\alpha$-condensing random operators, such that (i), (ii) (from Theorem 1) and

(iii) there exists $\rho \in(0, r)$ and $e \in K \backslash\{0\}$ such that for any

$$
\omega \in \Omega, x-f(\omega, x) \neq \lambda e \text { for }\|x\|=\rho \text { and } \lambda>0
$$

are satisfied.

Then $f$ has a random fixed point in $K_{\rho, r}$.

Proof: Let $P_{r}$ be as before and for all $\omega \in \Omega$,

$$
C(\omega)=\sup \left\{\|f(\omega, x)\|: x \in K_{r}\right\} .
$$

We use (ii) to get a "barrier" at $\|x\|=\rho$ as follows. Let $\phi_{n}: \Omega \times[0, r] \rightarrow[0, \infty)$ be a continuous random map such that $\phi_{n}(\omega, t)=0$ for $t \geq \rho$ and $\phi_{n}(\omega, t)=\delta(\omega)$ for $t \leq \rho-\frac{1}{n}$ and large $n$, with a measurable map $\delta: \Omega \rightarrow(0, \infty)$ such that $\delta(\omega)\|e\|>\rho+C(\omega)$ for each $\omega$. Let $f_{n}(\omega, x)=$ $\left(f(\omega, \cdot) \circ P_{r}\right) x+\phi_{n}(\omega,\|x\|)$. Evidently, $f_{n}$ is $\alpha$-condensing and weakly inward random operator on $K$. By Theorem 1, there exists a measurable map $\zeta_{n}: \Omega \rightarrow K$ such that $\zeta_{n}(\omega)=$ $f_{n}\left(\omega, \zeta_{n}(\omega)\right)$ for each $\omega \in \Omega$. Fix $\omega \in \Omega$ arbitrarily. We cannot have $\left\|\zeta_{n}(\omega)\right\|>r$. Hence, $\zeta_{n}(\omega)=f\left(\omega, \zeta_{n}(\omega)\right)+\phi_{n}\left(\omega,\left\|\zeta_{n}(\omega)\right\|\right)$. By the choice of $\phi_{n}$ we cannot have $\left\|\zeta_{n}(\omega)\right\| \leq \rho-\frac{1}{n}$, and we are done if $\left\|\zeta_{n}(\omega)\right\| \geq \rho$. So assume that $\rho-\frac{1}{n}<\left\|\zeta_{n}(\omega)\right\|<\rho$ for all large $n$. Since $\left\{\phi_{n}\left(\omega,\left\|\zeta_{n}(\omega)\right\|\right)\right\}$ is bounded and $f$ is $\alpha$-condensing, we have without loss of generality $\zeta_{n}(\omega)-$ 
$f\left(\omega, \zeta_{n}(\omega)\right) \rightarrow \lambda e$ and $\zeta_{n}(\omega) \rightarrow \zeta_{0}(\omega)$ with $\left\|\zeta_{0}(\omega)\right\|=\rho$ where $\lambda$ depends on $\omega$. Hence, $\zeta_{0}(\omega)=$ $f\left(\omega, \zeta_{0}(\omega)\right)+\lambda e$ and therefore $\lambda=0$ by $(i i i)$.

Theorem 3: Let $X$ be a separable Banach space, $K \subset X$ a cone such that $K_{1}$ is not compact, $f: \Omega \times K_{r} \rightarrow X$ a compact random operator satisfying $(i)$ and (ii) of Theorem 1 and

(iv) there exists $\rho \in(0, r)$ such that $f(\omega, x) \neq \lambda x$ for $\|x\|=\rho$ and $\lambda \in(0,1)$ and $\inf \{\|f(\omega, x)\|:\|x\|=\rho\}>0$, for each $\omega \in \Omega$.

Then $f$ has a random fixed point in $K_{\rho, r}$.

Proof: Since for any $\omega \in \Omega \inf \{\|f(\omega, x)\|:\|x\|=\rho\}>0$, and $A=\{x \in K:\|x\|=1\}$ is not compact, we can find $e \in A$ such that $-\lambda e \notin \overline{f(\omega, \rho A)}$ for all $\lambda \geq 0$.

Consider a random operator $f_{n}: \Omega \times K \rightarrow X$ defined by

$$
f_{n}(\omega, x)=\left\{\begin{array}{cc}
\left(f(\omega, \cdot) \circ P_{r}\right) x & \text { for }\|x\| \geq \rho \\
f\left(\omega, \frac{\rho}{\|x\|} x\right) & \text { for } 0<\rho_{0} \leq\|x\|<\rho \\
\frac{\|x\|}{\rho_{0}} f\left(\omega, \frac{\rho}{\|x\|} x\right)+\phi_{n}(\omega,\|x\|) e & \text { for }\|x\|<\rho_{0}
\end{array}\right.
$$

where $\phi_{n}: \Omega \times\left[0, \rho_{0}\right] \rightarrow[0, \infty)$ is a continuous random map, $\phi_{n}\left(\omega, \rho_{0}\right)=0$ and $\phi_{n}(\omega, t)=\delta(\omega)$ for $t \leq \rho_{0}\left(1-\frac{1}{n}\right)$, with a measurable map $\delta: \Omega \rightarrow(0, \infty)$ such that

$$
\delta(\omega)>\rho_{0}+\sup \{\|f(\omega, x)\|:\|x\|=\rho\} \text { for each } \omega \in \Omega
$$

Since $f_{n}$ is compact and a weakly inward random operator, it has a random fixed point $\xi_{n}: \Omega \rightarrow$ $K_{r}$ as before. We cannot have $\rho_{0} \leq\left\|\xi_{n}(\omega)\right\|<\rho$ or $\left\|\xi_{n}(\omega)\right\| \leq \rho_{0}\left(1-\frac{1}{n}\right)$ by $(i v)$ and the choice of $\phi_{n}$. If, for any $\omega \in \Omega,\left\|\xi_{n}(\omega)\right\| \geq \rho$, then we are done. Now assume that there exists $\omega$ such that $\rho_{0}-\frac{1}{n}<\left\|\zeta_{n}(\omega)\right\|<\rho_{0}$ for all large $n$. Then

$$
\frac{\left\|\xi_{n}(\omega)\right\|}{\rho} \eta_{n}(\omega)-\frac{\left\|\xi_{n}(\omega)\right\|}{\rho_{0}} f\left(\omega, \eta_{n}(\omega)\right)+\phi_{n}\left(\omega,\left\|\xi_{n}(\omega)\right\|\right) e
$$

with $\left\|\eta_{n}(\omega)\right\|=\left\|\frac{\rho}{\left\|\xi_{n}(\omega)\right\|} \xi_{n}(\omega)\right\|=\rho$. By letting $n \rightarrow \infty$ we get $\frac{\rho_{0}}{\rho} y_{0}=f\left(\omega, y_{0}\right)+\lambda e$ with $y_{0} \in \rho A$ and $0 \leq \lambda \leq \delta(\omega)$ depending on $\omega$, hence $\rho_{0} \rightarrow 0$ yields $-\lambda_{0} e \in \overline{f(\omega, \rho A)}$ for some $\lambda_{0}>0$, contradicting the choice of $e$.

Remark 4: Theorem 3 does not hold if $K_{1}$ is compact. For a counterexample, see [3].

\section{Acknowledgement}

This work is partially supported by Kuwait University Research Grant No. SM-108.

\section{References}

[1] Beg, I. and Shahzad, N., Random fixed points of random multivalued operators on Polish spaces, J. Nonlinear Anal. 20 (1993), 835-847.

[2] Bharucha-Reid, A.T., Fixed point theorems in probabilistic analysis, Bull. Amer. Math. Soc. 82 (1976), 641-657.

[3] Deimling, K., Positive fixed points of weakly inward maps, J. Nonlinear Anal. 12 (1988), 223-226. 
[4] Itoh, S., Random fixed point theorems with an application to random differential equations in Banach spaces, J. Math. Anal. Appl. 67 (1979), 261-273.

[5] Lin, T.C., Random approximation and random fixed point theorems for non-self-maps, Proc. Amer. Math. Soc. 103:4 (1988), 1129-1135.

[6] Papageorgiou, N.S., Random fixed point theorems for measurable multifunctions in Banach spaces, Proc. Amer. Math. Soc. 97 (1986), 507-514.

[7] Sehgal, V.M. and Singh, S.P., On random approximations and a random fixed point theorem for set-valued mappings, Proc. Amer. Math. Soc. 95 (1985), 91-94.

[8] Sehgal, V.M. and Waters, C., Some random fixed point theorems for condensing operators, Proc. Amer. Math. Soc. 90 (1984), 425-429.

[9] Tan, K.K. and Yuan, X.Z., Random fixed point theorems and approximation, J. Stoch. Anal. Appl., (to appear).

[10] Xu, H.K., Some random fixed point theorems for condensing and nonexpansive operators, Proc. Amer. Math. Soc. 110 (1990), 395-400. 


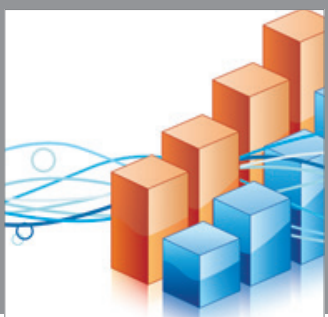

Advances in

Operations Research

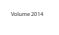

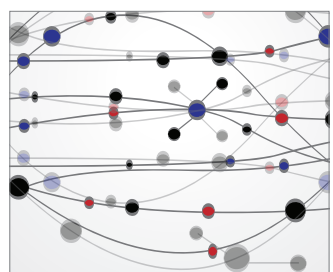

\section{The Scientific} World Journal
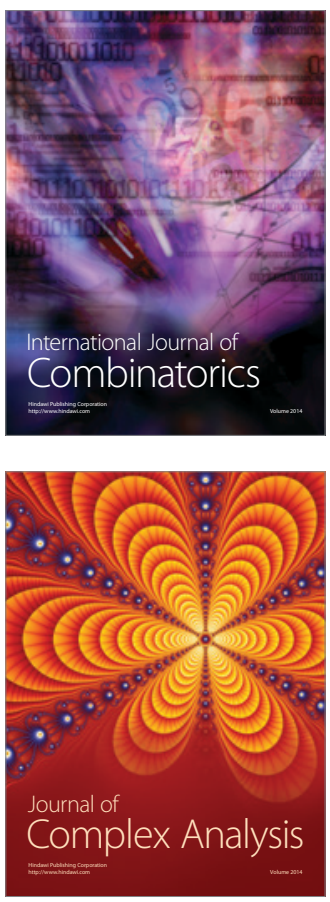

International Journal of

Mathematics and

Mathematical

Sciences
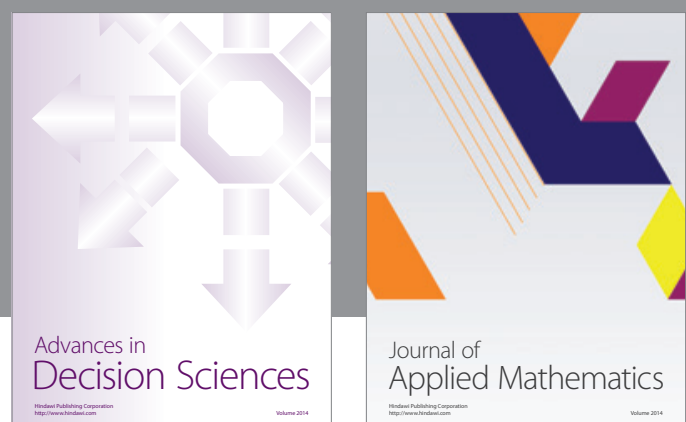

Journal of

Applied Mathematics
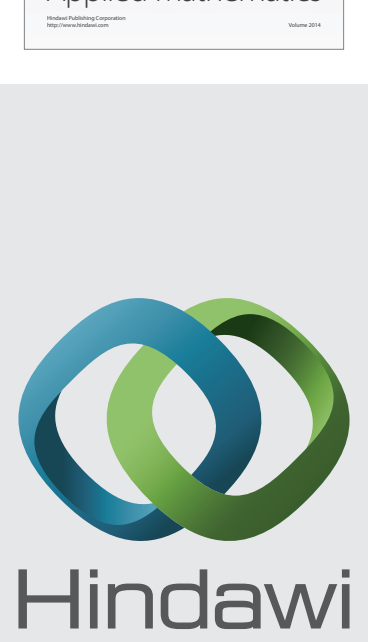

Submit your manuscripts at http://www.hindawi.com
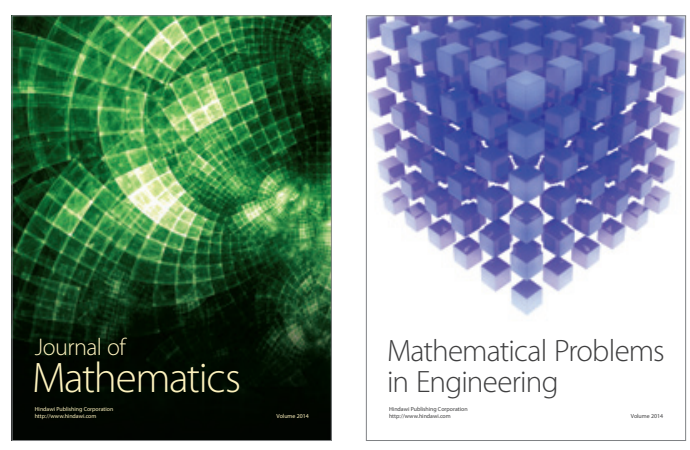

Mathematical Problems in Engineering
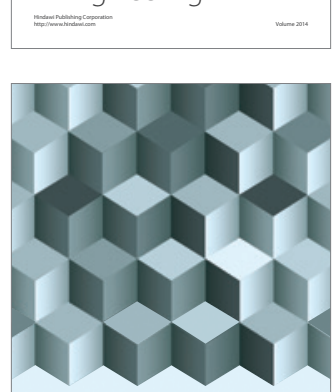

Journal of

Function Spaces
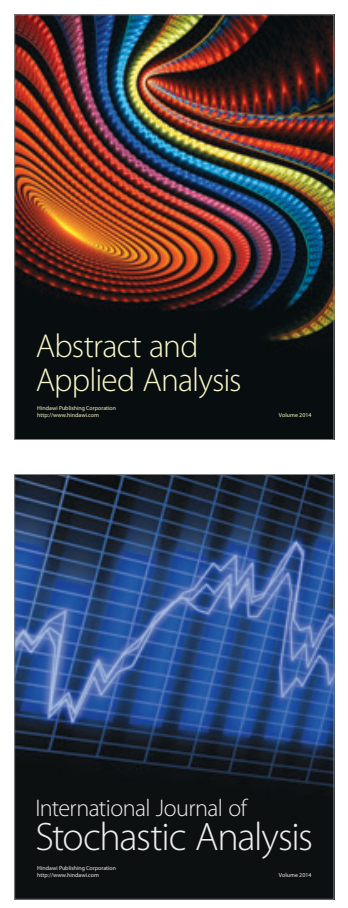

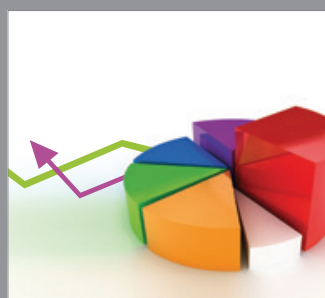

ournal of

Probability and Statistics

Promensencen
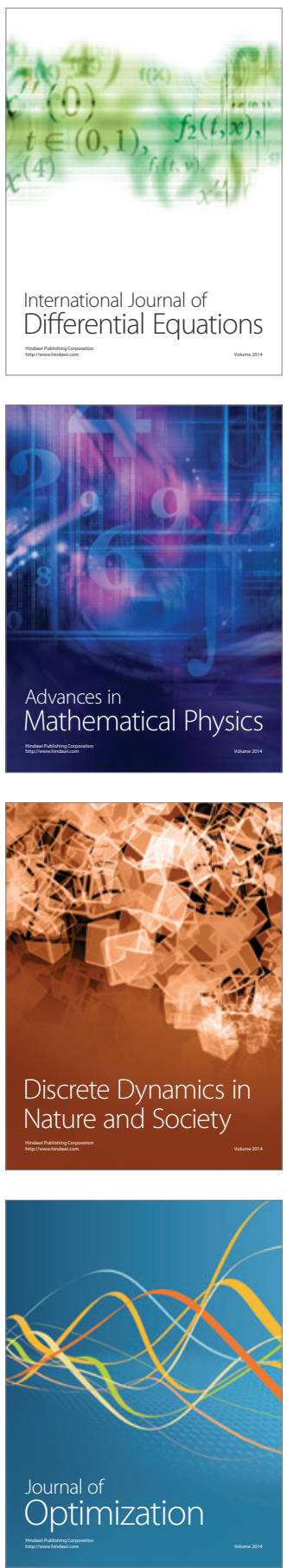